\title{
Optimization of the stand for test of hybrid rocket engines of solid fuel
}

\author{
Nikolay Zolotorev", Valeriy Kuznetsov, and Aleksey Konovalenko \\ National Research Tomsk State University, Tomsk, Russia
}

\begin{abstract}
In the paper the laboratory experimental stand of the hybrid rocket engine of solid fuel to study ballistic parameters of the engine at burning of high-energy materials in flow of hot gas is presented. Mixture of air with nitrogen with a specified content of active oxygen is used as a gaseous oxidizer. The experimental stand has modular design and consists of system of gas supply, system of heating of gas, system for monitoring gas parameters, to which a load cell with a model engine was connected. The modular design of the stand allows to change its configuration under specific objective. This experimental stand allows to conduct a wide range of the pilot studies at interaction of a hot stream of gas with samples highenergy materials.
\end{abstract}

\section{Introduction}

Experimental study of interaction of hot stream of gas with samples of high-energy materials (HEM) is used for projection of perspective rocket engines, and for experimental verification of the existing theoretical models and calculation data executed on their basis. In the course of experiment it is important to control not only thermodynamic and gasdynamic parameters of process (temperature, pressure, gas consumption) but also ballistic parameters (rocket engine thrust) [1].

\section{Experimental stand}

To carry out experimental studies based on the analysis of literature sources, the design of a laboratory stand for a hybrid rocket engine solid fuel (HRESF) was developed. Sample of HEM as is used the studied material with the central channel on the centre of which the gaseous oxidizer arriving from the block of preparation of gas mix is blown is used $[2,3]$. High rates of motion of the combustion products of the charge assume that not all fuel has time to react in the combustion chamber, in this regard HRESF is complemented by an afterburner [4-8].

The scheme of the experimental stand for research of burning of samples of high-energy materials in the hot gas stream is shown in figure 1.

\footnotetext{
* Corresponding author: nikzolotorev@mail.ru
} 


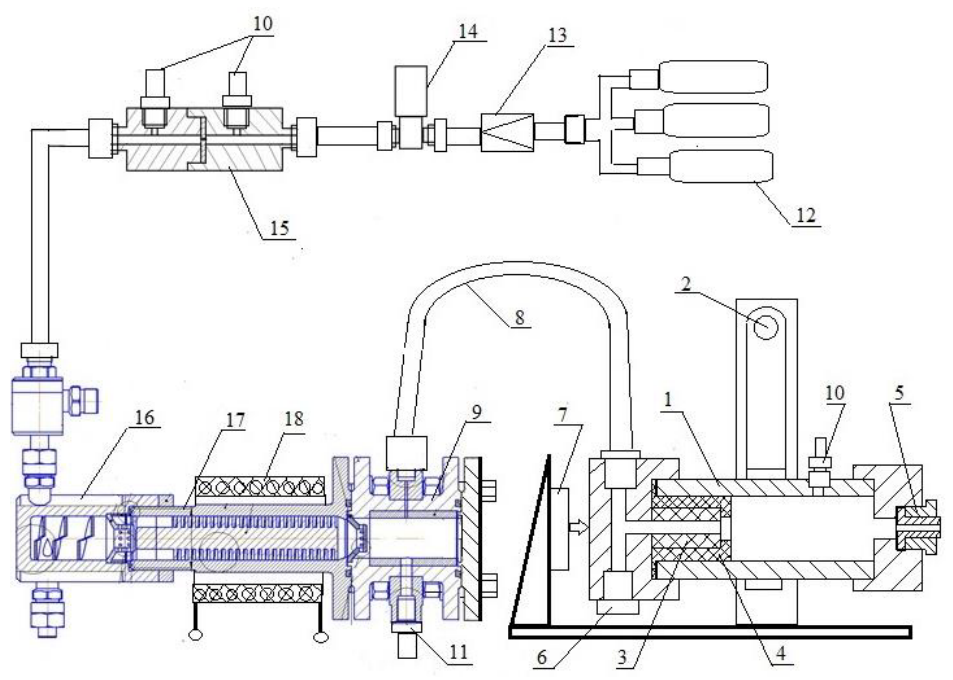

Fig. 1. The scheme of the experimental stand with heating of air. 1 - model engine; 2 - pendular suspender of the stand; 3 - sample of HEM; 4 - container for sample HEM; 5 - nozzle; 6, 10 pressure sensors; 7 - sensor thrust; 8 - flexible metal sleeve; 9 - antechamber; 11 - temperature sensor; 12 - cylinder with gas; 13 - reducer; 14 - electropneumatic valve; 15 - differential flowmeter; 16 - mixer; 17 - oven of heating of gas; 18 - inductor.

The experimental stand consists of two blocks: the block of preparation of gas mix and the measuring block executed in the form of the model rocket engine. The model rocket engine is installed at the stand for measuring the thrust. Air was used as the working gas in the experimental stand. This experimental stand allows using different mixes of gases by means of the mixer. Heating of gas is carried out in the labyrinth furnace by means of the $40 \mathrm{kVA}$ induction heater (furnace heating temperature to $900 \mathrm{~K}$ ). Gas parameters at the exit of the block of preparation of gas mix (in the antechamber) made: pressure is up to $0.3 \mathrm{MPa}$, temperature is up to $600 \mathrm{~K}$, gas consumption $5 \div 30 \mathrm{of} g / \mathrm{s}$.

The modular design of the stand allows changing its configuration under specific objective. For receiving hot gas stream with temperature over $550 \mathrm{~K}$ in installation use of solid-fuel gas generator which is established instead of the induction heater is provided. The scheme of the block of preparation of gas on the basis of solid-fuel gas generator is presented on figure 2 .

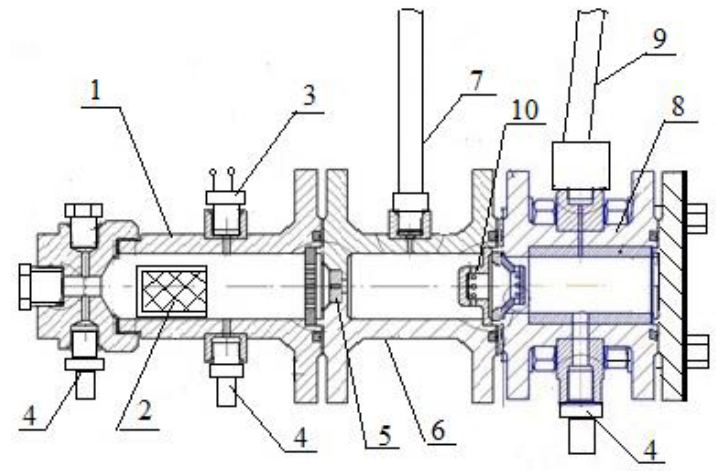

Fig. 2. Gas generator of solid-fuel with the camera of mixing and the antechamber. 1 - gas generator body; 2 - charge of solid-fuel; 3 - igniter; 4 - pressure sensor; 5 - nozzle; 6 - mixing camera; 7 - air supply; 8 - antechamber; 9 - supply gas mixture to a model engine; 10 - diaphragm. 
In this case between gas generator and the antechamber the camera of mixing of gases (figure 2) is installed $[9,10]$. Air for obtaining necessary temperature of gas mix and the set content of oxygen, moves in the mixing camera across gas stream from gas generator and mixes up, being filtered through system of diaphragms. As fuel for gas generator the charge of gunpowder of brand " $\mathrm{H}$ " was used. Temperature of products of burning of gunpowder " $\mathrm{H}$ " is $2368 \mathrm{~K}$. The gas rate made of gas generator $20 \div 80 \mathrm{of} \mathrm{g} / \mathrm{s}$ with depending on combustion chamber pressure.

The studied samples HEM in the form of cylindrical checker height $50 \mathrm{~mm}$ diameter $40 \mathrm{~mm}$ and the internal channel $10 \mathrm{~mm}$ were placed in the textolite container in front part of the model engine coaxially with the channel with a diameter of $10 \mathrm{~mm}$ of supply of gas mix. Burning of sample of HEM is carried out on the internal channel. The engine rigidly fastened in the pendulum suspender of the stand for measurement of thrust and front cover leaned on the thrust measurement sensor. Both blocks of the stand connected by means of the flexible metal pipeline of the bellows type providing free swing of pendulum.

Measurement of pressure at the stand was taken by means of tensometric LX-412 and MBS-3000 sensors. Intrinsic error of measurement of pressure for LX-412 sensors no more than $0.8 \%$, and for MBS-3000 sensors no more than $0.5 \%$. Temperature was taken by thermocouples of BP 5/20 with diameter of electrodes of $0.35 \mathrm{~mm}$. The thrust was measured using force sensors DST1909 and MLB20. The gas rate was measured on differential pressure on the calibrated diaphragm. As data acquisition system digital unit were used: tensometric AT1-8 amplifier and programmable SIMATIC S7-1200 controller. The tensometiric AT1-8 amplifier has sampling rate to $5 \mathrm{kHz}$, measurement error of rated input signal no more than $0.2 \%$ at nonlinearity of transformation no more than $0.1 \%$. Accuracy of transformation of analog signal for the programmable SIMATIC S7-1200 controller makes $3 \%$. Signals from measuring apparatuses were transmitted to the personal computer and registered in the form of spreadsheets.

\section{Results of experimental study}

The temperatures and pressure received during experiment are shown in figure 3.

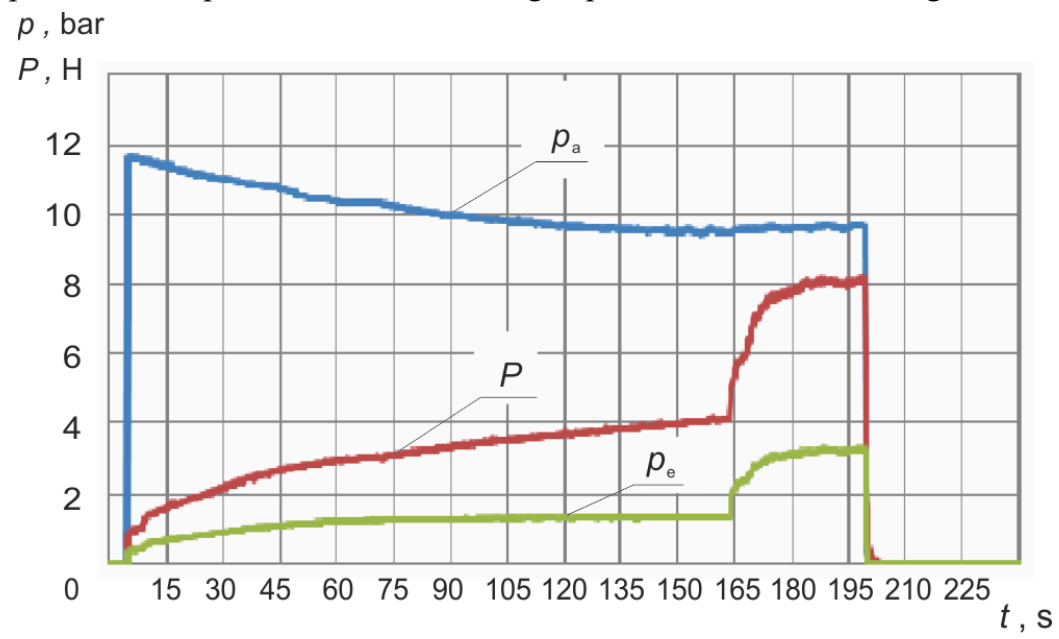




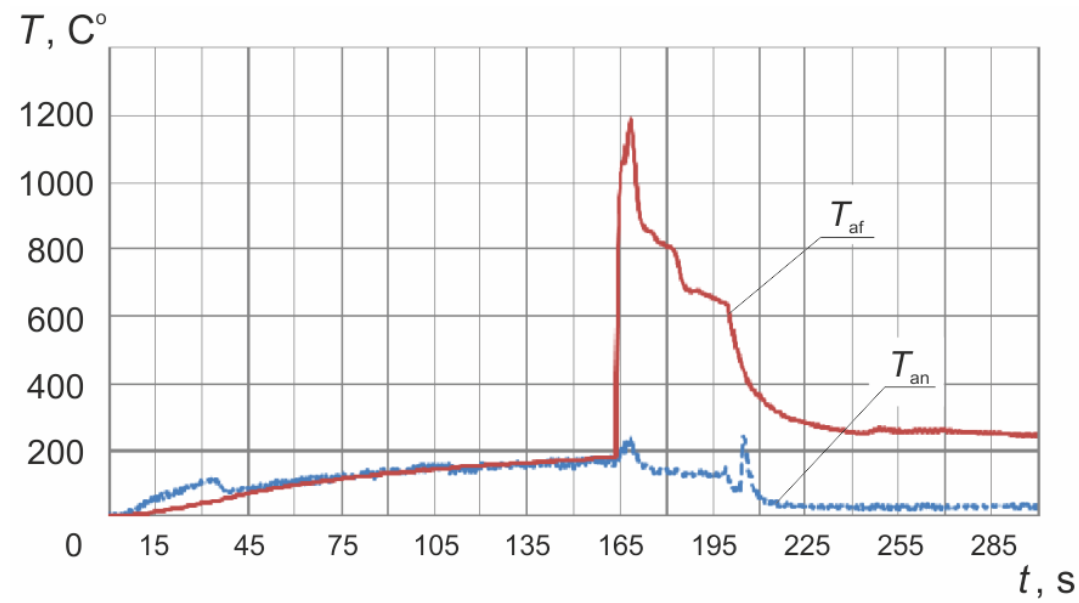

Fig. 3. Typical oscillograms of the process experimental study.

The data obtained from pressure sensors where $p_{\mathrm{a}}$ - pressure of air, $P$ - thrust of the model engine, $p_{\mathrm{e}}$ - the chamber pressure of afterburner of the model engine are presented on figure 3 . The data obtained from thermocouples $T_{\text {af }}-$ gas temperature in afterburner of the model engine, $T_{\mathrm{an}}$ - gas temperature in the antechamber are presented on figure 3 .

At air supply, temperature and gas pressure in afterburner of the model engine, and also its thrust gradually increase. After ignition of sample of HEM gas parameters and thrust of the engine sharply increase in the combustion camera. In process of burning out of heatedup layer parameters of gas and thrust of the model engine aim at quasistationary state. At air supply shutdown burning of sample of HEM stops both parameters of gas and thrust of the model engine sharply decrease. The appearance of the sample of HEM before and after blowing with hot gas stream, shown in figure 4.

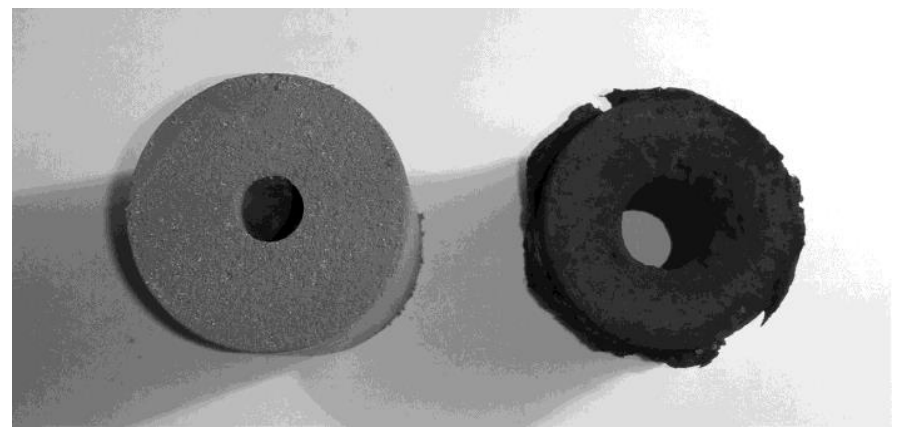

Fig. 4. Sample of HEM before tests at the stand (at the left) and after tests.

\section{Conclusions}

On the basis of the developed outline design documentation the HRESF laboratory stand is made. Test experimental studies of samples of HEM at the stand are conducted. Taking into account results of experimental studies testing of the software for calculation intra chamber and the HRESF power traction parameters is executed. The HRESF this laboratory stand allows providing carrying out experimental studies under different conditions of interaction of more hot gas stream with HEM samples. 
This work was supported financially by the Ministry of Education and Science of the Russian Federation in terms of state order, project code 9.9036.2017.

\section{References}

1. V.T. Volkov, D.A. Yagodnikov, Studies and bench testing of solid-propellant rocket engines (BMSTU Publ., Moscow, 2007)

2. D.A. Yagodnikov, A.V. Voronetskii, V.I. Sarab'ev, J. Combust. Expl. Shock Waves, 52, 300 (2016)

3. V.A. Arkhipov, S.S. Bondarchuk, A.B. Vorozhtsov, A.S. Zhukov, B.V. Pevchenko, L.A. Savelyeva, Patent Rus. No 2014149746 (2015)

4. J. Liu, D. Liang, J. Xiao, B. Chen, Ya. Zhang, J. Zhou, K. Cen, J. Combust. Expl. Shock Waves, 53, 55 (2017)

5. M.A. Karabeyoglu, D. Altman, B.J. Cantwell, J. Propul. Power, 18, 610 (2002)

6. M.A. Karabeyoglu, D. Altman, B.J. Cantwell, J. Propul. Power, 18, 621 (2002)

7. G.A. Marxman, M. Gilbert, Internat. Symp. on Combust., 9, 371 (1963)

8. V.A. Arkhipov, S.S. Bondarchuk, A.B. Vorozhtsov, L. De Luca, A.G. Korotkikh, L. Galfetti, G. Colombo, A. Bandera, Izv. Vyssh. Uchebn. Zaved., Fiz., 9/2, 3 (2007)

9. G.P. Sutton, O. Biblarz, Hybrid propellant rockets (Rocket Propul. Elements. John Wiley \& Sons, New York, 2001)

10. L.T. De Luca, Hybrid rocket engines (In: Energetic problems in aerospace Propulsion, Milano, 2009) 